\title{
Concurrent Chemoradiotherapy with Temozolomide Followed by Adjuvant Temozolomide for Newly Diagnosed Glioblastoma Patients: A Retrospective Multicenter Observation Study in Korea
}

\begin{tabular}{|c|c|}
\hline Byung Sup Kim, MD'1 & Ho Jun Seol, MD, PhD² \\
\hline Do-Hyun Nam, MD, PhD² & Chul-Kee Park, MD, PhD³ \\
\hline II Han Kim, MD, PhD ${ }^{4}$ & Tae Min Kim, MD, PhD ${ }^{5}$ \\
\hline Jeong Hoon Kim, MD, PhD ${ }^{6}$ & Young Hyun Cho, MD ${ }^{6}$ \\
\hline Sang Min Yoon, MD, PhD & Jong Hee Chang, MD, PhD ${ }^{8}$ \\
\hline Seok-Gu Kang, MD, PhD & Eui Hyun Kim, MD8 \\
\hline Chang-Ok Suh, MD, $\mathrm{PhD}^{9}$ & Tae-Young Jung, MD, PhD ${ }^{10}$ \\
\hline Kyung-Hwa Lee, MD, PhD ${ }^{11}$ & Chae-Yong Kim, MD, PhD'12 \\
\hline In Ah Kim, MD, PhD'13 & Chang-Ki Hong, MD ${ }^{14}$ \\
\hline Heon Yoo, MD, PhD ${ }^{15}$ & Jin Hee Kim, MD, PhD' \\
\hline Shin-Hyuk Kang, MD, PhD ${ }^{17}$ & Min Kyu Kang, MD'18 \\
\hline Eun-Young Kim, MD'19 & Sun-Hwan Kim, MD²0 \\
\hline Dong-Sup Chung, MD, PhD²1 & Sun-Chul Hwang, MD²2 \\
\hline Joon-Ho Song, MD²3 & Sung Jin Cho, MD, PhD²4 \\
\hline Sun-II Lee, MD, PhD²5 & Youn-Soo Lee, MD, PhD²6 \\
\hline Kook-Jin Ahn, MD, PhD² & Se Hoon Kim, MD, PhD 28 \\
\hline Do Hun Lim, MD, PhD 29 & Ho-Shin Gwak, MD, $\mathrm{PhD}^{30}$ \\
\hline Se-Hoon Lee, MD, $\mathrm{PhD}^{31}$ & Yong-Kil Hong, MD, $\mathrm{PhD}^{32}$ \\
\hline
\end{tabular}

*A list author's affiliations appears at the end of the paper.
Correspondence: Se-Hoon Lee, MD, PhD Division of Hematology/Oncology, Department of Internal Medicine, Samsung Medical Center, Sungkyunkwan University School of Medicine, 81 Irwon-ro, Gangnam-gu, Seoul 06351, Korea

Tel: $82-2-3410-1132$

Fax: 82-2-3410-1757

E-mail: sehoon.lee119@gmail.com

Co-correspondence: Ho-Shin Gwak, MD, PhD Department of Systemic Cancer Science,

Graduate School of Cancer Science and Policy,

National Cancer Center, 323 Ilsan-ro,

Ilsandong-gu, Goyang, 10408, Korea

Tel: 82-31-920-1666

Fax: 82-31-920-2798

E-mail:nsghs@ncc.re.kr

Received December 8, 2015

Accepted June 6, 2016

Published Online June 27, 2016

*Byung Sup Kim, Ho Jun Seol, and Do-Hyun Nam contributed equally to this work.

\section{Purpose}

The purpose of this study was to investigate the feasibility and survival benefits of combined treatment with radiotherapy and adjuvant temozolomide (TMZ) in a Korean sample.

\section{Materials and Methods}

A total of 750 Korean patients with histologically confirmed glioblastoma multiforme, who received concurrent chemoradiotherapy with TMZ (CCRT) and adjuvant TMZ from January 2006 until June 2011, were analyzed retrospectively.

Results

After the first operation, a gross total resection (GTR), subtotal resection (STR), partial resection (PR), biopsy alone were achieved in 388 (51.7\%), 159 (21.2\%), 96 (12.8\%), and 107 (14.3\%) patients, respectively. The methylation status of $0^{6}$-methylguanine-DNA methyltransferase (MGMT) was reviewed retrospectively in 217 patients. The median follow-up period was 16.3 months and the median overall survival (OS) was 17.5 months. The actuarial survival rates at the 1-, 3-, and 5-year OS were $72.1 \%, 21.0 \%$, and $9.0 \%$, respectively. The median progression-free survival (PFS) was 10.1 months, and the actuarial PFS at 1-, 3-, and 5-year PFS were 42.2\%, 13.0\%, and 7.8\%, respectively. The patients who received GTR showed a significantly longer OS and PFS than those who received STR, PR, or biopsy alone, regardless of the methylation status of the MGMT promoter. Patients with a methylated MGMT promoter also showed a significantly longer OS and PFS than those with an unmethylated MGMT promoter. Patients who received more than six cycles of adjuvant TMZ had a longer OS and PFS than those who received six or fewer cycles. Hematologic toxicity of grade 3 or 4 was observed in $8.4 \%$ of patients during the CCRT period and in $10.2 \%$ during the adjuvant TMZ period.

\section{Conclusion}

Patients treated with CCRT followed by adjuvant TMZ had more favorable survival rates and tolerable toxicity than those who did not undergo this treatment.

Key words

Glioblastoma, Temozolomide, MGMT, Chemoradiotherapy 


\section{Introduction}

Glioblastoma multiforme (GBM) is the most common malignant type of primary brain tumor, comprising approximately $52 \%$ of all primary brain tumors [1]. Despite the use of a multidisciplinary treatment approach, the prognosis of GBM is extremely poor; most patients die within 2 years after the initial diagnosis [2-4]. Currently, the standard treatment for newly diagnosed GBM consists of a surgical resection to the extent feasible, followed by concurrent chemoradiotherapy with temozolomide (CCRT) and adjuvant temozolomide (TMZ). In 2004, a randomized phase III multicenter trial reported that CCRT followed by adjuvant TMZ improved the median survival and 2-year survival over postoperative radiotherapy (RT) alone [5]. Based on these results, the U.S. Food and Drug Administration approved the concomitant use of TMZ with RT for newly diagnosed GBM patients. Through a follow-up study longer than 5 years, Stupp et al. [6] reported that CCRT followed by adjuvant TMZ improved the long-term survival compared to RT alone, and that methylation of the $\mathrm{O}^{6}$-methylguanine-DNA methyltransferase (MGMT) promoter was correlated with a better overall survival (OS). Recently, Yang et al. [7] reported a variance in the correlations between MGMT promoter methylation and both the OS and progression-free survival (PFS) among an ethnically-diverse sample of GBM patients. In their metaanalysis, they found that subjects of European descent had a better OS and PFS in GBM with the MGMT promoter methylation compared to different race according to univariate or multivariate analysis, whereas subjects of East Asian descent had a longer OS compared to difference race according to multivariate analysis only. Only a few studies of OS and PFS among Korean GBM patients that account for MGMT promoter methylation have been reported. In particular, there are no large case studies of Korean GBM patients examining the efficacy and safety of CCRT followed by adjuvant TMZ.

This study assessed the efficacy and safety of CCRT followed by adjuvant TMZ for patients with a newly diagnosed GBM through a multicenter, retrospective study of a large case series for the first time in Korea.

\section{Materials and Methods}

\section{Patient sample}

Patients older than 20 years with a newly diagnosed GBM were assessed through retrospective chart reviews. All patients were confirmed histologically with GBM. Nineteen institutions participated in the study, and chart reviews for the clinical and radiological findings were performed independently and analyzed at each institution. The institutional review boards of all participating centers approved the study. A central pathologic review of histopathological specimens from all patients included in the study was performed by three neuropathologists (K.-H.L., Y.-S.L., and S.H.K.).

\section{Treatment protocols}

All patients were treated with CCRT with or without adjuvant TMZ according to Stupp's regimens [5]. The maximal surgical resection of tumors was performed within the feasible limits. The extent of the resection was classified as a gross total resection (GTR), subtotal resection (STR), partial resection $(\mathrm{PR})$, or biopsy alone. Patients received CCRT within 6 weeks after the pathological confirmation of GBM where possible. The patients were irradiated with a total dose of $60 \mathrm{~Gy}$, with daily fractions of 2 Gy given 5 days per week for 6 weeks, using conventional three-dimensional conformal RT. Alternative RT protocols, such as two-dimensional RT and intensity-modulated RT with or without a simultaneous-integrated boost were included to avoid any selection bias and reflect regular clinical situations. TMZ was administered at $75 \mathrm{mg} / \mathrm{m}^{2}$ every day for 6 weeks during the RT period. After a 4-week break, the patients received adjuvant TMZ for the first 5 days of each 28-day cycle provided that they continued to tolerate the treatment. The dose of adjuvant TMZ was $150 \mathrm{mg} / \mathrm{m}^{2} /$ day for the first cycle, which was increased to $200 \mathrm{mg} / \mathrm{m}^{2} /$ day at the beginning of the second cycle if no hematologic toxicity had occurred.

\section{Follow-up and evaluations}

The extent of the resection was evaluated by postoperative brain magnetic resonance imaging (MRI) with gadolinium enhancement within 48 hours after surgery. The GTR was defined as $>99 \%$ removal of the initial tumor, STR as $50 \%$ $99 \%$, and the PR as $<50 \%$. The MRI scans were performed before the first cycle and after every two or three cycles of adjuvant TMZ. The assessments of the radiologic outcomes were defined according to the RANO criteria [8]. The primary end-point was the OS, and the secondary end-points were PFS and safety. The PFS was defined as the time from the date of the initial operation to documented disease progression or the date of the final follow-up visit. The OS time was measured from the date of the initial operation to death or the date of the final follow-up visit. Patients who were alive at the time of analysis were included as censored observations. The methylation status of the MGMT promoter was evaluated using a methylation-specific polymerase chain reaction where possible. The toxic effects of the treatment 
A

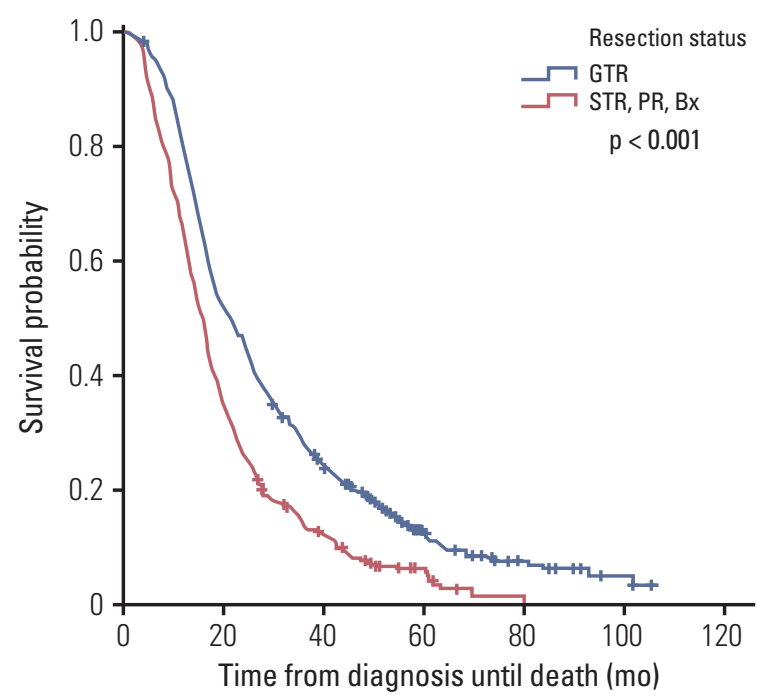

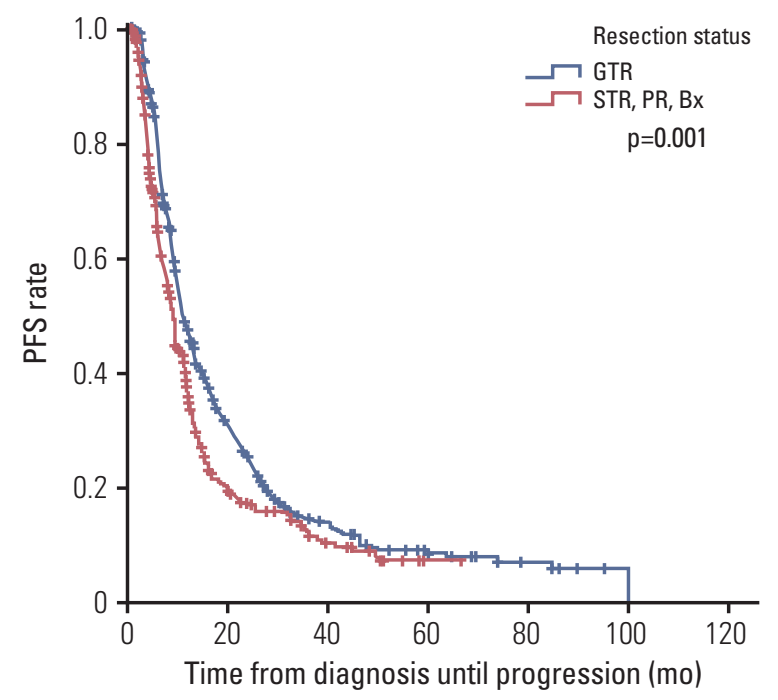

Fig. 1. Kaplan-Meier curves showing overall survival (OS) (A) and progression-free survival (PFS) (B) according to the extent of the resection. Patients who received gross total resection (GTR) showed a significantly longer OS (21.0 months vs. 15.8 months) and PFS (10.9 months vs. 9.1 months) than those who received subtotal resection (STR), partial resection (PR), or biopsy (Bx) alone.

were graded as follows according to the National Cancer Institute Common Terminology Criteria for Adverse Events (NCI CTCAE) ver. 4.0.3: a score of 1 , indicating mild adverse effects; 2, indicating moderate adverse effects; 3 , indicating severe adverse effects; 4 , indicating life-threatening adverse effects; and 5, denoting death related to adverse effects.

\section{Statistical analysis}

The prognostic factors associated with survival, including the age at diagnosis, sex, preoperative Karnofsky performance status (KPS), tumor location, extent of resection, use of adjuvant TMZ, and methylation status of the MGMT promoter, were examined by univariate and multivariate analyses. The OS and PFS were analyzed using the Kaplan-Meier method with two-sided log-rank tests. Differences were considered statistically significant at $\mathrm{p}$-value $<0.05$. All statistical analyses were performed using SPSS ver. 23.0 (IBM Corp., Armonk, NY).

\section{Results}

\section{Patient characteristics}

A total of 750 patients from 19 institutions were enrolled and treated according to Stupp's protocol from January 2006 until June 2011. Of these patients, $415(55.3 \%)$ were male and $335(44.7 \%)$ were female; the median age at the first operation was 57.5 years (range, 20 to 86 years). Three hundred eightyseven patients (51.7\%) showed a KPS score $\geq 90$ at diagnosis. Supratentorial lesions were observed in 719 patients (95.9\%) and infratentorial lesions were noted in 31 patients (4.1\%). Among the supratentorial lesions, deep-seated GBMs were found in 106 patients (4.7\%) and superficial GBMs were found in 613 patients $(85.3 \%)$. At the first operation, GTR, STR, PR, and biopsy alone were achieved in 388 (51.7\%), 159 $(21.2 \%), 96(12.8 \%)$, and $107(14.3 \%)$ patients, respectively. A central pathological review confirmed the diagnosis of GBM in all cases reviewed. The median time from diagnosis to the start of RT was 3.6 weeks (range, 0.7 to 26.6 weeks). The median therapeutic radiation dose was $60 \mathrm{~Gy}$ (range, 4 to 84 Gy). Among the 750 patients assigned to receive CCRT, 659 $(87.9 \%)$ completed CCRT as planned. After CCRT, 660 patients $(88 \%)$ started adjuvant TMZ and received a median of six cycles (range, 0 to 15 cycles); 407 patients (61.7\%) completed six cycles. The reasons for not starting or not complet- 

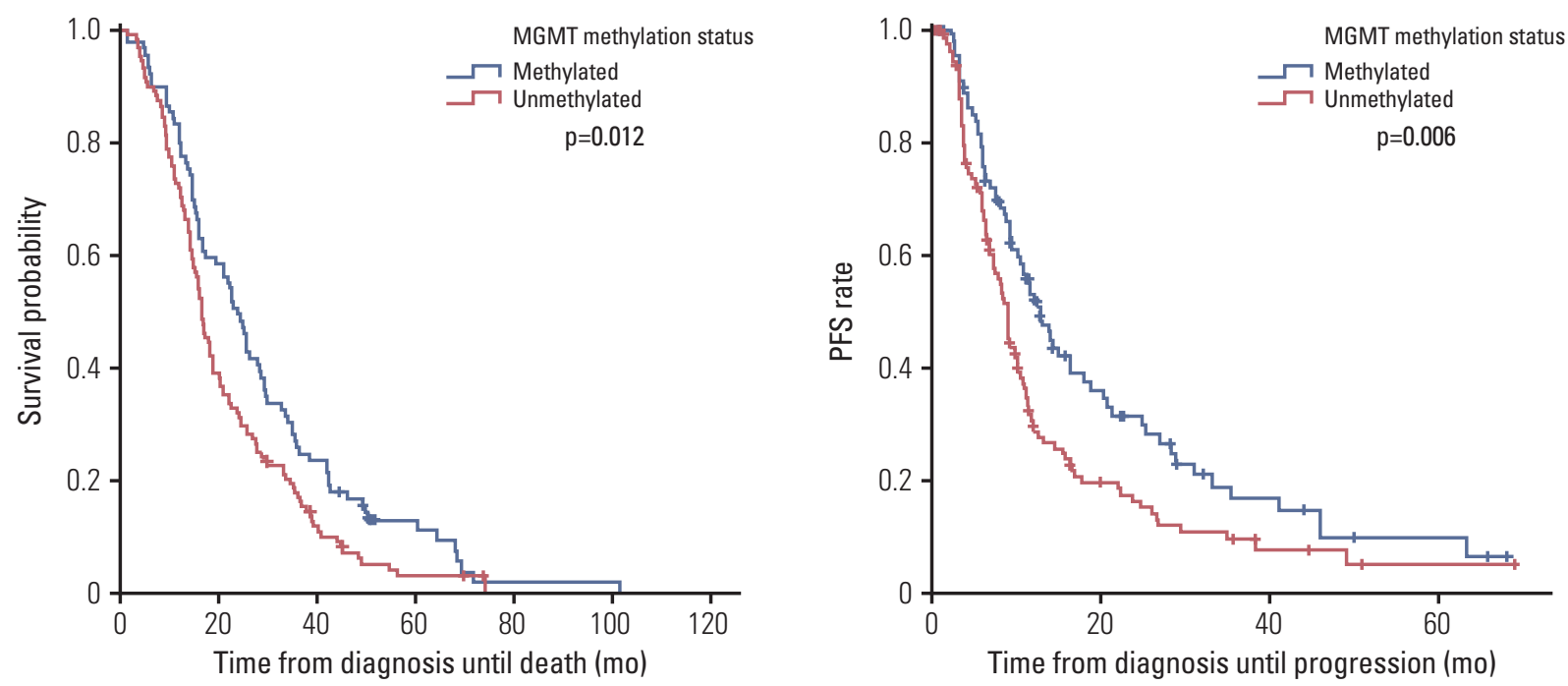

Fig. 2. Kaplan-Meier curves showing overall survival (OS) (A) and progression-free survival (PFS) (B) according to the $\mathrm{O}^{6}{ }_{-}$ methylguanine-DNA methyltransferase (MGMT) promoter methylation status. Patients with the methylated MGMT promoter had a longer OS (23.9 months vs. 16.7 months) and longer PFS (13.2 months vs. 9.3 months) than those with the unmethylated MGMT promoter.

ing adjuvant TMZ therapy were disease progression (32.6\%), toxic effects $(3.5 \%)$, and follow-up loss $(2.3 \%)$. The median KPS in those patients who completed and did not complete adjuvant TMZ was 90 (range, 20 to 100) and 80 (30 to 100), respectively. The methylation status of the MGMT promoter was evaluated in 217 of the 750 patients (28.9\%). Of the 217 patients, $89(41.0 \%)$ had the methylated MGMT promoter, and $128(59.0 \%)$ had the unmethylated MGMT promoter.

\section{Survival outcome and progression}

At the time of the analysis, 690 patients $(92.0 \%)$ had died. The median follow-up period was 16.3 months (range, 0.3 to 105.1 months), and the median OS was 17.5 months (95\% confidence interval [CI], 16.5 to 18.5). The actuarial survival rates at 1-, 3-, and 5-year OS were $72.1 \%, 21.0 \%$, and $9.0 \%$, respectively. The median PFS was 10.1 months $(95 \%$ CI, 9.3 to 10.9), and the actuarial PFS at 1-, 3-, and 5-year PFS was $42.2 \%, 13.0 \%$, and $7.8 \%$, respectively. In univariate analysis for survival in all patients, the age at diagnosis $(\mathrm{p}<0.001)$, preoperative KPS $(p<0.001)$, tumor location $(p<0.001)$, extent of resection ( $\mathrm{p}<0.001)$, use of adjuvant TMZ ( $\mathrm{p}<0.001)$, and methylation status of the MGMT promoter $(\mathrm{p}=0.012)$ were correlated with survival. The age at diagnosis $(\mathrm{p}=0.003)$, extent of the resection $(p<0.001)$, and use of adjuvant TMZ $(p<0.001)$ were correlated with the survival in both univariate and multivariate analyses. In addition, the methylation status of the MGMT promoter ( $\mathrm{p}=0.083$ ) was not correlated with survival in multivariate analysis. Patients who had received GTR showed a significantly longer OS (21.0 months vs. 15.8 months, $\mathrm{p}=0.001$ ) (Fig. $1 \mathrm{~A}$ ) and longer PFS (10.9 months vs. 9.1 months, $\mathrm{p}=0.001$ ) (Fig. 1B) than those who had received STR, PR, or biopsy alone in both univariate and multivariate analyses. Patients with the methylated MGMT promoter had a longer OS (23.9 months vs. 16.7 months, $\mathrm{p}=0.012$ ) (Fig. 2A) and longer PFS (13.2 months vs. 9.3 months, $\mathrm{p}=0.006$ ) (Fig. 2B) than those with the unmethylated MGMT promoter. When analyzing the extent of the resection according to the methylation status of the MGMT, patients receiving GTR showed a significantly longer OS than those receiving STR, PR, or biopsy alone in the groups with both the methylated (28.6 months vs. 16.7 months, $\mathrm{p}=0.001$ ) (Fig. 3A) and unmethylated MGMT promoter (19.0 months vs. 14.8 months, $\mathrm{p}=0.001$ ) (Fig. $3 \mathrm{C}$ ). As with OS, a significantly higher PFS was observed in patients receiving GTR compared to those receiving STR, PR, or biopsy alone in both groups with the methylated (20.7 months vs. 11.1 months, $\mathrm{p}=0.041$ ) (Fig. 3B) and unmethylated MGMT promoter (10.6 months vs. 7.2 months, $\mathrm{p}=0.030$ ) (Fig. 3D). The use of adjuvant TMZ was associated with survival according to both univariate and multivariate analyses. The OS and PFS were compared in patients with the methylated and unmethylated MGMT promoter, considering the degree of completeness of adjuvant TMZ (Table 1). Patients with the methylated 
A
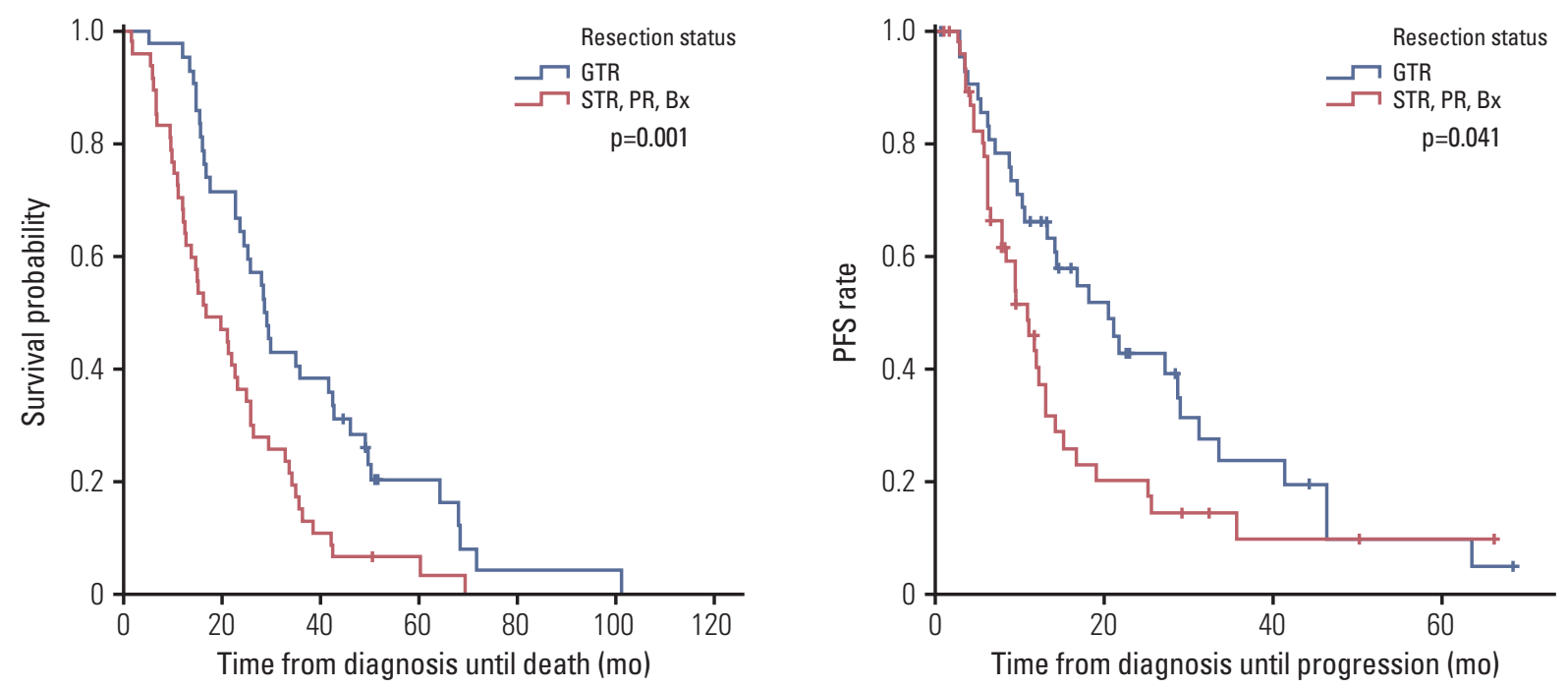

C
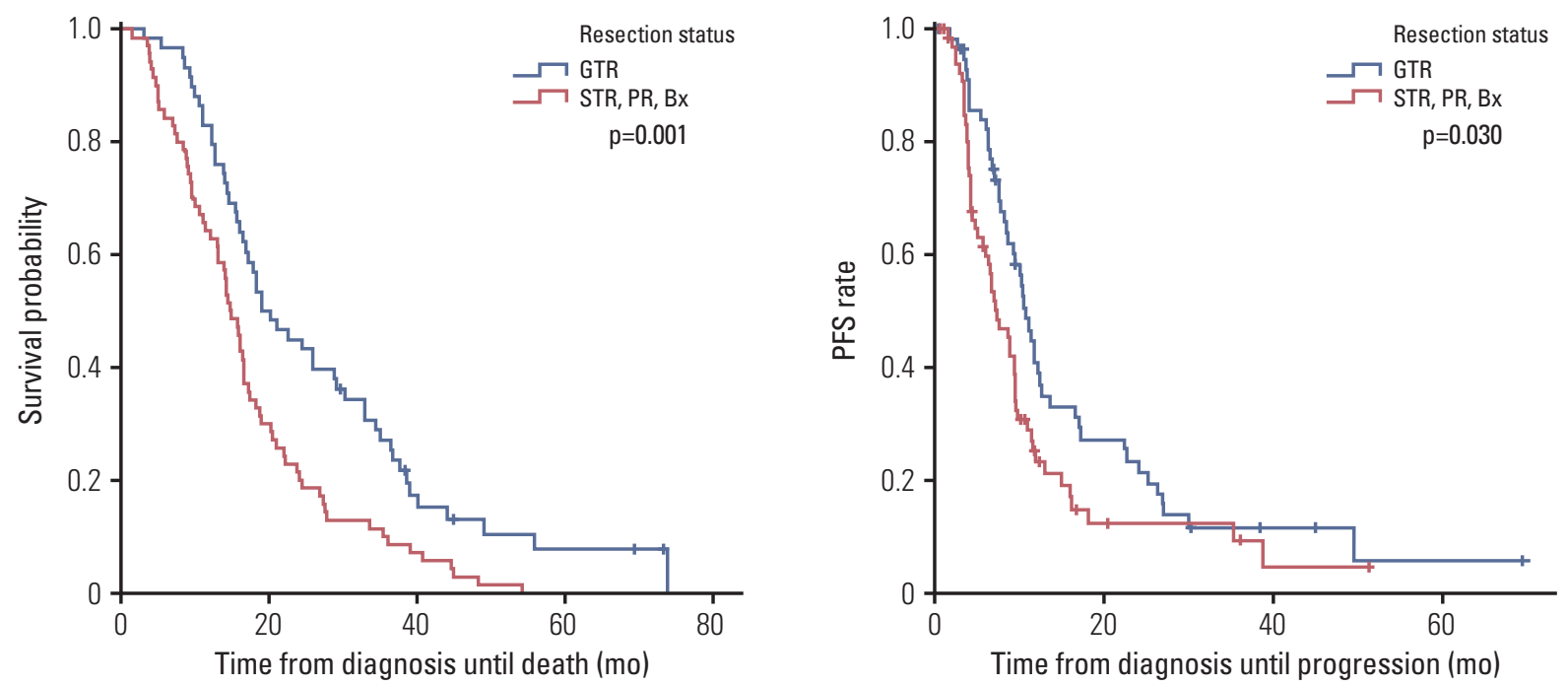

Fig. 3. Kaplan-Meier curves showing overall survival (OS) (A) and progression-free survival (PFS) (B) according to the extent of the resection in patients with the methylated $\mathrm{O}^{6}$-methylguanine-DNA methyltransferase (MGMT) promoter. KaplanMeier curves showing OS (C) and PFS (D) according to the extent of resection in patients with unmethylated MGMT promoter. Patients receiving gross total resection (GTR) demonstrated a significantly longer OS than those receiving subtotal resection (STR), partial resection (PR), or biopsy (Bx) alone in both groups with methylated (28.6 months vs. 16.7 months) and unmethylated MGMT promoter (19.0 months vs. 14.8 months). For OS, patients receiving GTR demonstrated a significantly longer PFS than those receiving STR, PR, or Bx alone in both groups with methylated (20.7 months vs. 11.1 months) and unmethylated MGMT promoter (10.6 months vs. 7.2 months). 


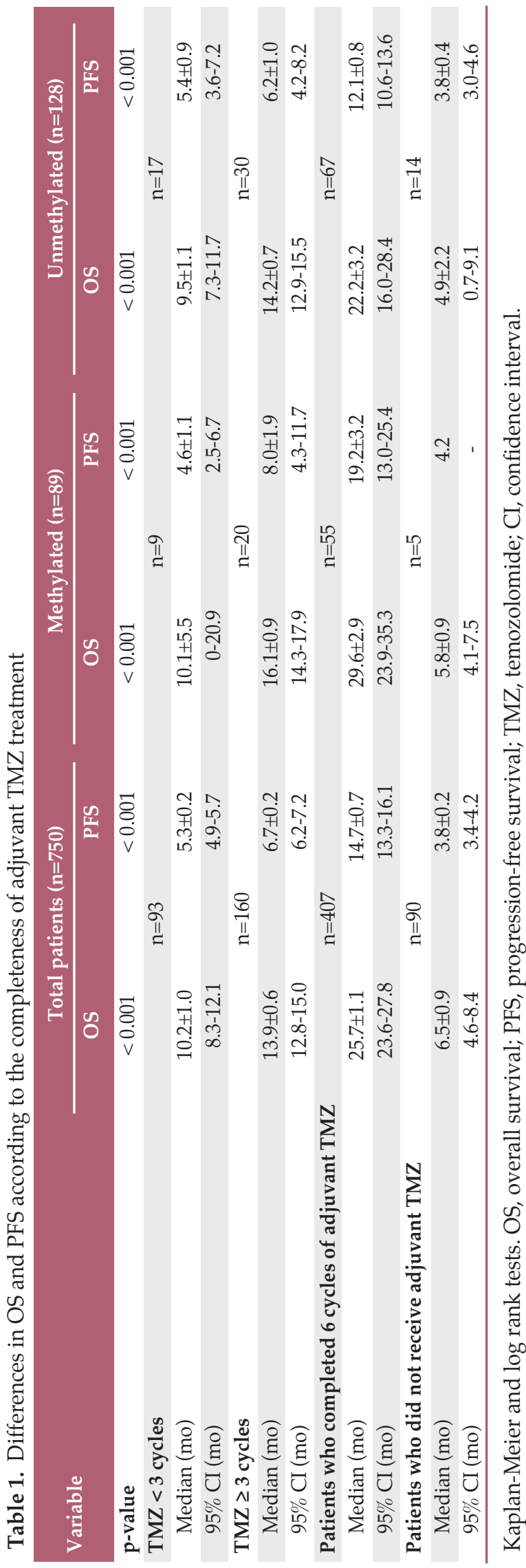

MGMT promoter had a longer OS and longer PFS as the number of adjuvant TMZ cycles approached six compared to those who had the unmethylated MGMT promoter (Fig. 4).

\section{Treatment-related hematologic toxicity}

The incidence and degree of hematologic toxicity were analyzed separately according to the period of CCRT and adjuvant TMZ. A total of 115 patients $(15.3 \%)$ showed grade 3 or 4 hematologic toxicity, as evaluated by CTCAE ver. 4.0.3 during the CCRT and the adjuvant TMZ period. During the CCRT period, grade 3 or 4 hematologic toxicity was noted in 63 patients $(8.4 \%)$, including 41 patients $(5.5 \%)$ with lymphocytopenia, 10 patients $(1.3 \%)$ with neutropenia, eight patients $(1.1 \%)$ with thrombocytopenia, seven patients $(0.9 \%)$ with leukopenia, and five patients $(0.7 \%)$ with anemia. During the adjuvant TMZ period, grade 3 or 4 hematologic toxicity was reported in 66 patients $(10.2 \%)$, including 42 patients $(6.4 \%)$ with lymphocytopenia, 17 patients $(2.6 \%)$ with neutropenia, 13 patients $(2.0 \%)$ with thrombocytopenia, five patients $(0.8 \%)$ with anemia, and two patients $(0.3 \%)$ with leukopenia.

\section{Discussion}

In previous studies, age, performance status, extent of resection, methylation status of the MGMT promoter, and the use of TMZ were identified as prognostic factors for patients with GBM $[6,9,10]$. In the present study, age, extent of the resection, and use of adjuvant TMZ were correlated with a better OS according to univariate and multivariate analyses, whereas the tumor location and methylation status of the MGMT promoter were prognostic factors of OS in univariate analysis alone. These findings are consistent with those of previous studies. Table 2 summarizes the results of Stupp's trial and other studies in East Asian samples.

In 2004, the European Organization for Research and Treatment of Cancer (EORTC) and National Cancer Institute of Canada (NCIC) trial (Stupp's trial) demonstrated a median OS and PFS of 14.6 and 6.9 months in patients with newly diagnosed and histologically proven GBM, respectively. In the present study, the median OS and PFS were 17.5 and 10.1 months, respectively. Although the treatment in the present study was based on Stupp's protocol, better clinical outcomes were observed than those of Stupp's trial. Several differences were observed between the studies, which could explain the improved survival. One possible explanation was the superior GTR rate. The number of TMZ-treated patients who received GTR was larger than that in Stupp's trial 
A
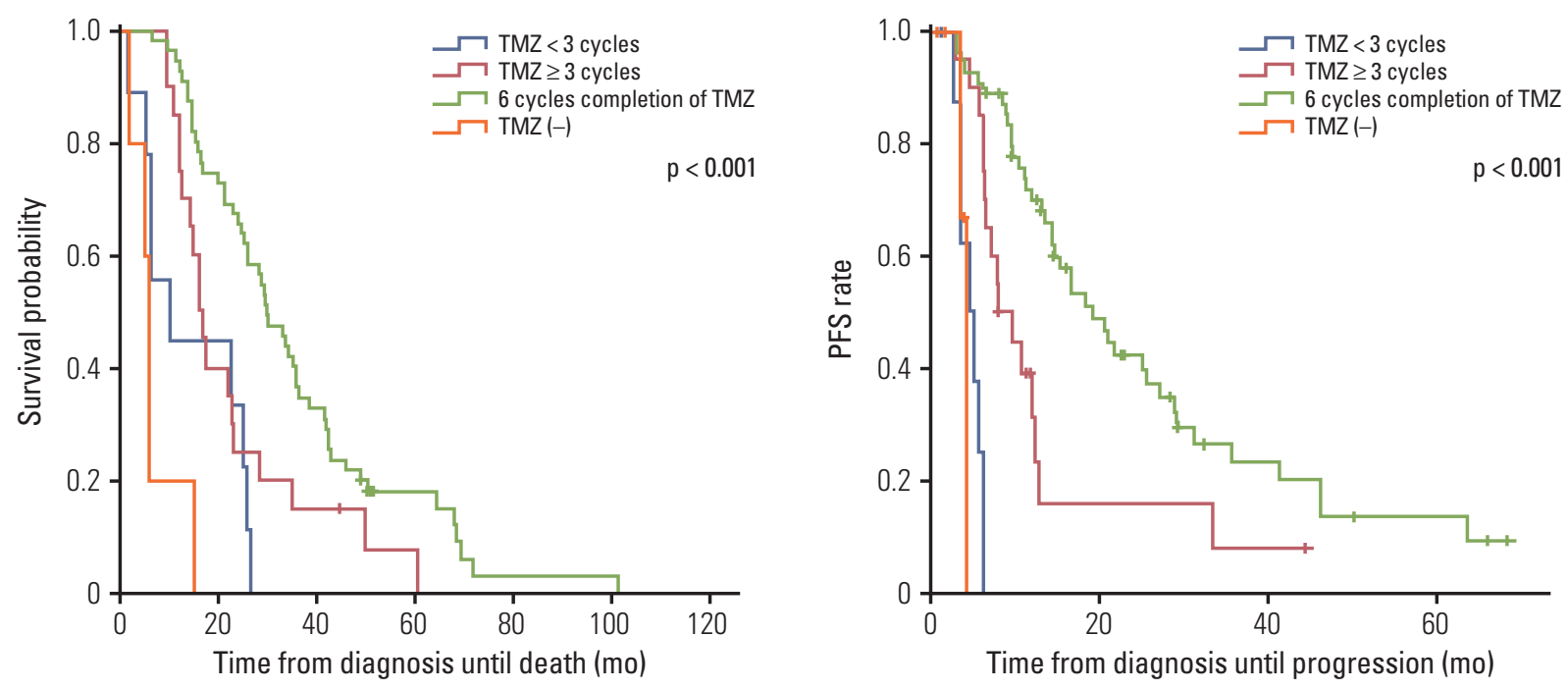

C
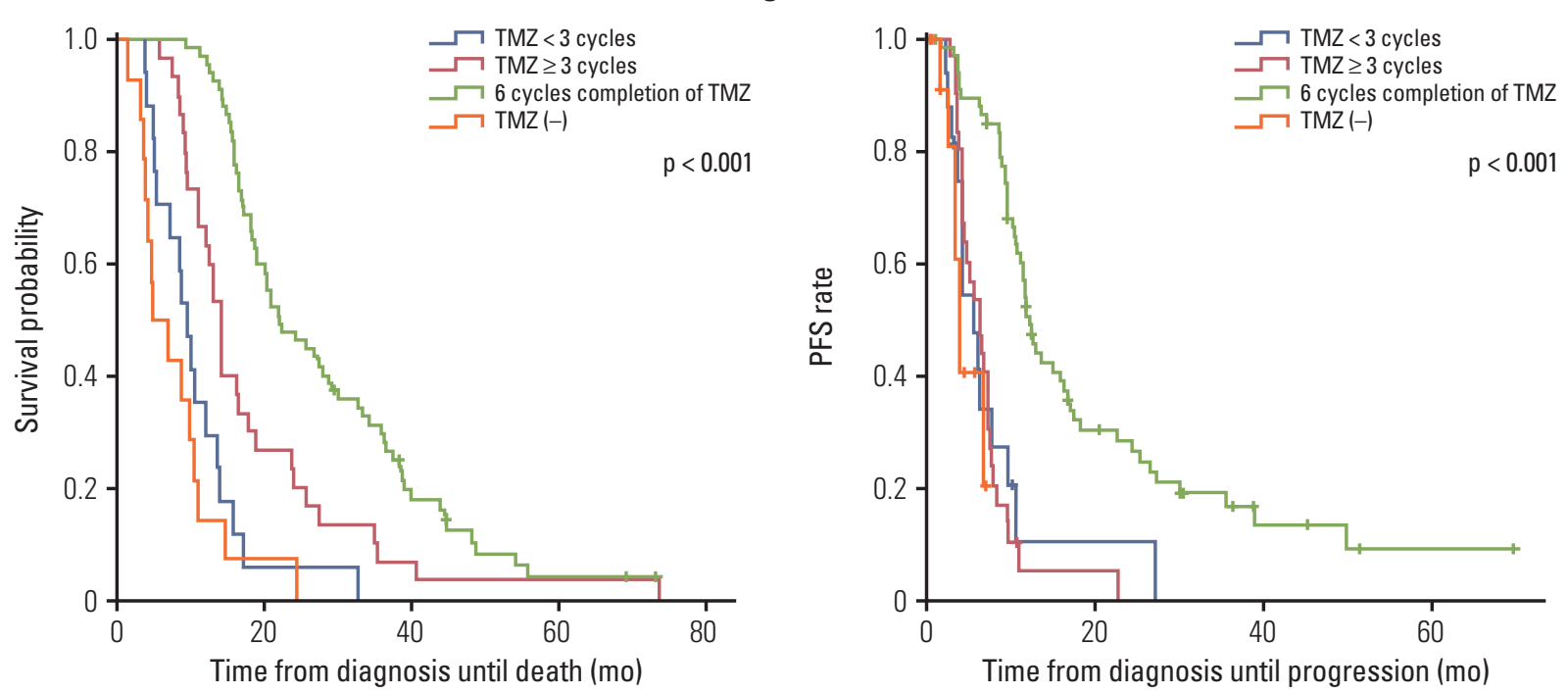

Fig. 4. Kaplan-Meier curves showing overall survival (OS) (A) and progression-free survival (PFS) (B) according to the completion of six cycles of adjuvant temozolomide (TMZ) in patients with the methylated $\mathrm{O}^{6}$-methylguanine-DNA methyltransferase (MGMT) promoter. Kaplan-Meier curves showing OS (C) and PFS (D) according to completion of six cycles of adjuvant TMZ in patients with the unmethylated MGMT promoter. Patients with the methylated MGMT promoter had a longer OS and longer PFS as the number of adjuvant TMZ cycles approached six compared to those with the unmethylated MGMT promoter.

(51.7\% vs. 39\%). The literature indicates that GTR is a significant prognostic factor in patients with a primary GBM [11-15]. In the current series, patients who received GTR showed a longer OS and longer PFS than those who received STR, PR, or biopsy alone, regardless of the methylation status of the MGMT promoter. Selection bias may also have occurred due to the differences between a prospective randomized study (Stupp's trial) and a retrospective multiinstitutional study (the present study).

The methylation status of the MGMT promoter is a suggested predictive factor of the survival of patients with newly diagnosed GBM treated with RT and TMZ [6,16,17]. The epi- 


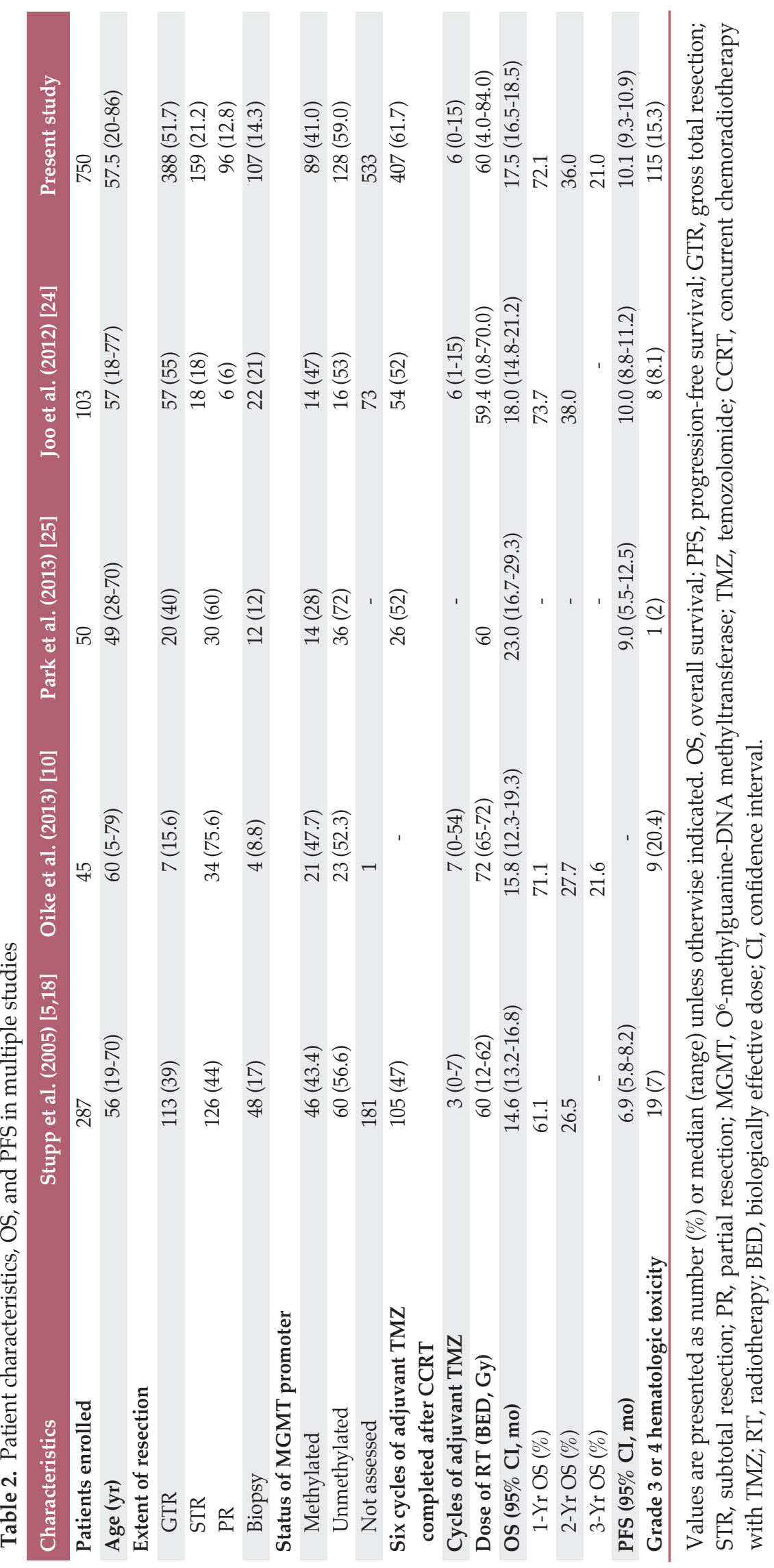


genetic silencing of the MGMT gene by methylation of the MGMT promoter leads to a loss of MGMT expression and a decrease in DNA repair activity, resulting in increased sensitivity to TMZ and a longer OS [5]. On the other hand, whether methylation of the MGMT promoter is truly a prognostic marker or a predictive marker of the sensitivity to alkylating agents or radiation is unclear [18-20]. In the current study, the MGMT methylation status was a prognostic factor of survival in univariate analysis alone. Patients with a methylated MGMT promoter and underwent Stupp's protocol showed significantly a longer OS and longer PFS than those with the unmethylated MGMT promoter. These results are consistent with those of a previous study [18]. Among the 573 patients enrolled in Stupp's trial, Hegi et al. [18] analyzed the MGMT methylation status in 206 patients for whom the MGMT status could be evaluated. In 106 patients who received CCRT, patients with methylated MGMT promoter $(43.4 \%)$ showed a significantly longer OS and longer PFS than those with the unmethylated MGMT promoter (56.6\%) (OS, 21.7 months vs. 12.7 months; PFS, 10.3 months vs. 5.3 months). These results also imply that a methylated MGMT promoter is predictive of a response to RT and is a prognostic factor in GBM.

The differences in survival between the present and previous studies could also be explained by the early start of postoperative RT and the number of adjuvant TMZ cycles administered. The median cellular doubling time of GBM is 17 days. As the tumor grows and produces satellite growths, the chance of a regional miss increases during radiation treatment [21]. Consequently, any delays in time from surgery to the start of RT are correlated with a poor survival [22]. The median time from surgery to the start of RT was 5 weeks in Stupp's trial, whereas postoperative RT was started within a median time of 3.5 weeks in the present study. A disparity in the degree of completion of adjuvant TMZ was observed between the groups. Although adjuvant TMZ was administered for a maximum of six cycles in Stupp's trial, in the present study, adjuvant TMZ was continued provided that it was tolerated (median cycle, 3 cycles vs. 6 cycles, respectively). The number of patients completing six cycles of adjuvant TMZ was also greater in the present study than in Stupp's trial (61.7\% vs. $47 \%)$. Seiz et al. [23] compared the OS and PFS in patients who received six cycles or fewer and more than six cycles of adjuvant TMZ. Among a total of 114 patients treated with Stupp's protocol, 55 received adjuvant TMZ of six cycles or less, and 59 received more than six cycles. Correlations were observed between the degree of completion of adjuvant TMZ and both OS and PFS [23]. In the present study, the administration of adjuvant TMZ after CCRT was the strongest prognostic factor of survival, and the degree of completion of adjuvant TMZ was correlated with the OS and PFS regardless of the methylation status of the MGMT pro- moter. These results suggest that adjuvant TMZ is crucial for prolonging the OS and PFS in Korean patients with a newly diagnosed GBM.

Finally, the incidence of hematologic toxicity of grade 3 or 4 during the CCRT period was slightly greater in the present study than in Stupp's trial (8.4\% vs. 6.7\%). In contrast to the CCRT period, the incidence of hematologic toxicity of grade 3 or 4 during the adjuvant TMZ period was lower in the present study than in Stupp's trial (10.2\% vs. $14.3 \%$ ). Hematologic toxicity of grade 3 or 4 was considered tolerable compared to other studies $[5,10,24,25]$.

\section{Conclusion}

Patients treated with CCRT followed by adjuvant TMZ had more favorable survival rates and tolerable toxicity. On the other hand, the present study has limitations because of its retrospective nature, the study range was restricted to adult GBM, and the use of a different treatment protocol from that already recognized as the standard for newly diagnosed GBM. Nevertheless, this study is the first large, multicenter, observational study of GBM treatment in a sample of Korean patients. A randomized, prospective study of a large case series will be needed explore ways of improving the survival among Korean GBM patients.

\section{Conflicts of Interest}

Conflict of interest relevant to this article was not reported.

\section{Author Details}

${ }^{1}$ Department of Neurosurgery, Kosin University Gospel Hospital, Kosin University College of Medicine, Busan, ${ }^{2}$ Department of Neurosurgery, Samsung Medical Center, Sungkyunkwan University School of Medicine, Seoul, Departments of ${ }^{3}$ Neurosurgery and ${ }^{4}$ Radiation Oncology, ${ }^{5}$ Division of Hematology / Oncology, Department of Internal Medicine, Seoul National University Hospital, Seoul National University College of Medicine, Seoul, Departments of ${ }^{6}$ Neurosurgery and ${ }^{7}$ Radiation Oncology, Asan Medical Center, University of Ulsan College of Medicine, Seoul, Departments of ${ }^{8}$ Neurosurgery and ${ }^{9}$ Radiation Oncology, Brain Tumor Center, Yonsei University Health System, Seoul, Depart- 
ments of ${ }^{10}$ Neurosurgery and ${ }^{11}$ Pathology, Chonnam National University Hwasun Hospital, Hwasun, Departments of ${ }^{12}$ Neurosurgery and ${ }^{13}$ Radiation Oncology, Seoul National University Bundang Hospital, Seoul National University College of Medicine, Seongnam, ${ }^{14}$ Department of Neurosurgery, Gangnam Severance Hospital, Yonsei University College of Health Science, Seoul, ${ }^{15}$ Department of NeuroOncology Clinic, Center for Specific Organs Cancer, National Cancer Center Hospital, National Cancer Center, Goyang, ${ }^{16}$ Department of Radiation Oncology, Keimyung University Dongsan Medical Center, Keimyung University School of Medicine, Daegu, ${ }^{17}$ Department of Neurosurgery, Korea University Anam Hospital, Korea University College of Medicine, Seoul, ${ }^{18}$ Department of Radiation Oncology, Yeungnam University Medical Center, Yeungnam University College of Medicine, Daegu, ${ }^{19}$ Department of Neurosurgery, Inha University Hospital, Inha University School of Medicine, Incheon, ${ }^{20}$ Department of Neurosurgery, Chungnam National University Hospital, Chungnam National University School of Medicine, Daejeon, ${ }^{21}$ Department of Neurosurgery, Incheon St. Mary's Hospital, College of Medicine, The
Catholic University of Korea, Incheon, ${ }^{22}$ Department of Neurosurgery, Soonchunhyang University Bucheon Hospital, Bucheon, ${ }^{23}$ Department of Neurosurgery, Hallym University Sacred Heart Hospital, Anyang, ${ }^{24}$ Department of Neurosurgery, Soonchunhyang University Hospital, Seoul, ${ }^{25}$ Department of Neurosurgery, Inje University Haeundae Paik Hospital, Inje University College of Medicine, Busan, Departments of ${ }^{26}$ Pathology and ${ }^{27}$ Radiation, Seoul St. Mary's Hospital, College of Medicine, The Catholic University of Korea, Seoul, ${ }^{28}$ Department of Pathology, Research Institute of Radiological Science, Yonsei University College of Medicine, Seoul, ${ }^{29}$ Department of Radiation Oncology, Samsung Medical Center, Sungkyunkwan University School of Medicine, Seoul, ${ }^{30}$ Department of Systemic Cancer Science, Graduate School of Cancer Science and Policy, National Cancer Center, Goyang, ${ }^{31}$ Division of Hematotology / Oncology, Department of Internal Medicine, Samsung Medical Center, Sungkyunkwan University School of Medicine, Seoul, ${ }^{32}$ Department of Neurosurgery, Seoul St. Mary's Hospital, College of Medicine, The Catholic University of Korea, Seoul, Korea

\section{References}

1. Suzuki Y, Shirai K, Oka K, Mobaraki A, Yoshida Y, Noda SE, et al. Higher pAkt expression predicts a significant worse prognosis in glioblastomas. J Radiat Res. 2010;51:343-8.

2. DeAngelis LM. Brain tumors. N Engl J Med. 2001;344:114-23.

3. Buckner JC. Factors influencing survival in high-grade gliomas. Semin Oncol. 2003;30(6 Suppl 19):10-4.

4. Curran WJ Jr, Scott CB, Horton J, Nelson JS, Weinstein AS, Fischbach AJ, et al. Recursive partitioning analysis of prognostic factors in three Radiation Therapy Oncology Group malignant glioma trials. J Natl Cancer Inst. 1993;85:704-10.

5. Stupp R, Mason WP, van den Bent MJ, Weller M, Fisher B, Taphoorn MJ, et al. Radiotherapy plus concomitant and adjuvant temozolomide for glioblastoma. N Engl J Med. 2005;352: 987-96.

6. Stupp R, Hegi ME, Mason WP, van den Bent MJ, Taphoorn MJ, Janzer RC, et al. Effects of radiotherapy with concomitant and adjuvant temozolomide versus radiotherapy alone on survival in glioblastoma in a randomised phase III study: 5-year analysis of the EORTC-NCIC trial. Lancet Oncol. 2009;10:45966.

7. Yang H, Wei D, Yang K, Tang W, Luo Y, Zhang J. The prognosis of MGMT promoter methylation in glioblastoma patients of different race: a meta-analysis. Neurochem Res. 2014;39:2277-87.

8. Wen PY, Macdonald DR, Reardon DA, Cloughesy TF, Sorensen AG, Galanis E, et al. Updated response assessment criteria for high-grade gliomas: response assessment in neurooncology working group. J Clin Oncol. 2010;28:1963-72.
9. Lamborn KR, Chang SM, Prados MD. Prognostic factors for survival of patients with glioblastoma: recursive partitioning analysis. Neuro Oncol. 2004;6:227-35.

10. Oike T, Suzuki Y, Sugawara K, Shirai K, Noda SE, Tamaki T, et al. Radiotherapy plus concomitant adjuvant temozolomide for glioblastoma: Japanese mono-institutional results. PLoS One. 2013;8:e78943.

11. Senft C, Bink A, Franz K, Vatter H, Gasser T, Seifert V. Intraoperative MRI guidance and extent of resection in glioma surgery: a randomised, controlled trial. Lancet Oncol. 2011;12: 997-1003.

12. Stummer W, Reulen HJ, Meinel T, Pichlmeier U, Schumacher W, Tonn JC, et al. Extent of resection and survival in glioblastoma multiforme: identification of and adjustment for bias. Neurosurgery. 2008;62:564-76.

13. Lacroix M, Abi-Said D, Fourney DR, Gokaslan ZL, Shi W, DeMonte $\mathrm{F}$, et al. A multivariate analysis of 416 patients with glioblastoma multiforme: prognosis, extent of resection, and survival. J Neurosurg. 2001;95:190-8.

14. Laws ER, Parney IF, Huang W, Anderson F, Morris AM, Asher A, et al. Survival following surgery and prognostic factors for recently diagnosed malignant glioma: data from the Glioma Outcomes Project. J Neurosurg. 2003;99:467-73.

15. Kreth FW, Thon N, Simon M, Westphal M, Schackert G, Nikkhah $\mathrm{G}$, et al. Gross total but not incomplete resection of glioblastoma prolongs survival in the era of radiochemotherapy. Ann Oncol. 2013;24:3117-23.

16. Esteller M, Hamilton SR, Burger PC, Baylin SB, Herman JG. 
Inactivation of the DNA repair gene $\mathrm{O}^{6}$-methylguanine-DNA methyltransferase by promoter hypermethylation is a common event in primary human neoplasia. Cancer Res. 1999;59: 793-7.

17. Esteller M, Garcia-Foncillas J, Andion E, Goodman SN, Hidalgo OF, Vanaclocha V, et al. Inactivation of the DNArepair gene MGMT and the clinical response of gliomas to alkylating agents. N Engl J Med. 2000;343:1350-4.

18. Hegi ME, Diserens AC, Gorlia T, Hamou MF, de Tribolet N, Weller M, et al. MGMT gene silencing and benefit from temozolomide in glioblastoma. N Engl J Med. 2005;352:997-1003.

19. Rivera AL, Pelloski CE, Gilbert MR, Colman H, De La Cruz C, Sulman EP, et al. MGMT promoter methylation is predictive of response to radiotherapy and prognostic in the absence of adjuvant alkylating chemotherapy for glioblastoma. Neuro Oncol. 2010;12:116-21.

20. Criniere E, Kaloshi G, Laigle-Donadey F, Lejeune J, Auger N, Benouaich-Amiel A, et al. MGMT prognostic impact on glioblastoma is dependent on therapeutic modalities. J Neurooncol. 2007;83:173-9.
21. Lawrence YR, Blumenthal DT, Matceyevsky D, Kanner AA, Bokstein F, Corn BW. Delayed initiation of radiotherapy for glioblastoma: how important is it to push to the front (or the back) of the line? J Neurooncol. 2011;105:1-7.

22. Irwin C, Hunn M, Purdie G, Hamilton D. Delay in radiotherapy shortens survival in patients with high grade glioma. J Neurooncol. 2007;85:339-43.

23. Seiz M, Krafft U, Freyschlag CF, Weiss C, Schmieder K, Lohr $\mathrm{F}$, et al. Long-term adjuvant administration of temozolomide in patients with glioblastoma multiforme: experience of a single institution. J Cancer Res Clin Oncol. 2010;136:1691-5.

24. Joo JD, Chang JH, Kim JH, Hong YK, Kim YH, Kim CY. Temozolomide during and after radiotherapy for newly diagnosed glioblastomas: a prospective multicenter study of Korean patients. J Korean Neurosurg Soc. 2012;52:92-7.

25. Park CK, Lee SH, Kim TM, Choi SH, Park SH, Heo DS, et al. The value of temozolomide in combination with radiotherapy during standard treatment for newly diagnosed glioblastoma. J Neurooncol. 2013;112:277-83. 